\title{
Collective hydrogen-bond rearrangement dynamics in liquid water
}

Cite as: J. Chem. Phys. 149, 244504 (2018); https://doi.org/10.1063/1.5054267

Submitted: 30 August 2018 . Accepted: 29 November 2018 . Published Online: 27 December 2018

R. Schulz, Y. von Hansen, J. O. Daldrop, J. Kappler (iD, F. Noé (D), and R. R. Netz (D)
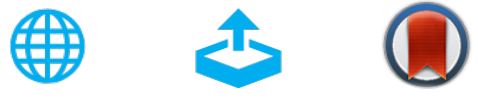

\section{ARTICLES YOU MAY BE INTERESTED IN}

Perspective: Computational chemistry software and its advancement as illustrated through three grand challenge cases for molecular science

The Journal of Chemical Physics 149, 180901 (2018); https://doi.org/10.1063/1.5052551

Common microscopic structural origin for water's thermodynamic and dynamic anomalies The Journal of Chemical Physics 149, 224502 (2018); https://doi.org/10.1063/1.5055908

Aqueous solvation from the water perspective

The Journal of Chemical Physics 148, 234505 (2018); https://doi.org/10.1063/1.5034225

Where in the world is AIP Publishing?

Find out where we are exhibiting next 


\title{
Collective hydrogen-bond rearrangement dynamics in liquid water
}

\author{
R. Schulz, ${ }^{1}$ Y. von Hansen, ${ }^{1}$ J. O. Daldrop,${ }^{1}$ J. Kappler, ${ }^{1}$ F. Noé,${ }^{2}$ and R. R. Netz ${ }^{1, a)}$ \\ ${ }^{1}$ Department of Physics, Freie Universität Berlin, 14195 Berlin, Germany \\ ${ }^{2}$ Department of Mathematics and Computer Science, Freie Universität Berlin, 14195 Berlin, Germany
}

(Received 30 August 2018; accepted 29 November 2018; published online 27 December 2018)

\begin{abstract}
We investigate barrier-crossing processes corresponding to collective hydrogen-bond rearrangements in liquid water using Markov state modeling techniques. The analysis is based on trajectories from classical molecular dynamics simulations and accounts for the full dynamics of relative angular and separation coordinates of water clusters and requires no predefined hydrogen bond criterium. We account for the complete 12-dimensional conformational subspace of three water molecules and distinguish five well-separated slow dynamic processes with relaxation times in the picosecond range, followed by a quasi-continuum spectrum of faster modes. By analysis of the Markov eigenstates, these processes are shown to correspond to different collective interchanges of hydrogen-bond donors and acceptors. Using a projection onto hydrogen-bond states, we also analyze the switching of one hydrogen bond between two acceptor water molecules and derive the complete transition network. The most probable pathway corresponds to a direct switch without an intermediate, in agreement with previous studies. However, a considerable fraction of paths proceeds along alternative routes that involve different intermediate states with short-lived alternative hydrogen bonds or weakly bound states. Published by AIP Publishing. https://doi.org/10.1063/1.5054267
\end{abstract}

\section{INTRODUCTION}

Water plays a key role for many biological, chemical, and physical processes. ${ }^{1}$ On the microscopic level, water dynamics can be described by the formation and breaking of hydrogen bonds (H-bonds) which is important for understanding protein, ${ }^{2,3}$ ligand-receptor, ${ }^{4}$ protein-surface, ${ }^{5}$ electrolyte, ${ }^{6,7}$ proton, ${ }^{8-10}$ and hydrophobic solvation dynamics. ${ }^{11,12}$ Despite the fundamental importance of the molecular dynamics (MD) in liquid water, the understanding of collective water restructuring events, such as the switching of a single $\mathrm{H}$ bond from one accepting water molecule to another, remains challenging. ${ }^{13,14}$

The classical view introduced by Debye ${ }^{15}$ describes the reorientation dynamics of a water molecule as a diffusion process. According to this model, when an H-bond is broken, the water molecule performs an angular Brownian motion until it finds a new H-bond partner. Although this view provides a straightforward interpretation of the water dielectric relaxation in the $\mathrm{GHz}$ regime, ${ }^{6,7}$ it was challenged based on two theoretical advancements: First, employing transition path sampling in conjunction with classical molecular dynamics simulations, it was suggested that in roughly half of the $\mathrm{H}$ bond breaking events, a new H-bond with a different water molecule is formed right after, ${ }^{16}$ partly confirming Stillinger's switching-of-allegiance scenario of the local hydrogen bonding dynamics. ${ }^{17}$ Second, it has been shown that the switch of an H-bond donor occurs typically through a rather abrupt

Note: This article is part of the Special Topic "Markov Models of Molecular Kinetics" in J. Chem. Phys.

a)Author to whom correspondence should be addressed: rnetz@physik.fuberlin.de angular jump, ${ }^{14,18,19}$ which is supported experimentally by neutron scattering ${ }^{20,21}$ and 2D-IR spectroscopy. ${ }^{22,23}$ In particular, it was demonstrated that the H-bond switch is a cooperative process that involves at least three water molecules. ${ }^{14,18,19}$ The prevalence of abrupt rotations suggests that non-H-bonded configurations, also referred to as dangling H-bonds, are freeenergetically unstable and therefore should only appear as short-lived transient states that either rebond with its initial $\mathrm{H}$-bond partner or quickly engage in a new H-bond with a different partner, which indeed has been demonstrated in simulations and experiments. ${ }^{24}$

We note that an abrupt angular change is not necessarily in conflict with Debye's diffusive model since the presence of an angular free energy barrier quite naturally predicts a transition path time that is much shorter than the H-bond lifetime ${ }^{25-27}$ (see Sec. III for more details on this). Experimentally, H-bond network rearrangements can be studied by femtosecond pump-probe IR spectroscopy and are characterized by time scales in the sub-picosecond to picosecond range, ${ }^{28-31} \mathrm{THz}$ absorption studies reflect $\mathrm{H}$-bond rearrangements in the sub-picosecond range, ${ }^{32}$ while photon echo peak shift studies demonstrate the existence of relaxation phenomena in the $5-15$ ps range. ${ }^{33}$ So based on the experimental results, $\mathrm{H}$-bond restructuring relaxation times span a broad range from a few picoseconds down to hundreds of femtoseconds. This wide range of experimental time scales of liquid water is reflected by an entire relaxation time spectrum of water rearrangement processes revealed by our analysis.

Similar questions about the kinetics of H-bond rearrangements also arise in isolated low-temperature water clusters, ${ }^{34-36}$ where concerted rotations of water molecules have been observed experimentally and have been interpreted in 
terms of cooperative H-bond switching. Clearly, in these low-temperature systems, the dynamics is governed by quantum tunneling, not by thermally activated barrier crossing. Due to the small number of involved molecules and the absence of stochastic effects in these systems, the possible pathways can for small clusters be enumerated exactly. ${ }^{35,36}$

From these low-temperature studies, one would conclude that also in bulk liquid water, structural rearrangements are collective and involve more than two water molecules. But water bonding kinetics in liquid water poses two additional problems that arise from the coupling to a dynamic finitetemperature environment. First, a priori it is not clear what minimum number of water molecules needs to be considered to meaningfully describe dynamic restructuring of the liquid. Second, the stochastic coupling to a random liquid environment creates a multitude of thermally activated competing pathways that connect given initial and final water structures of a barrier-crossing process.

In principle, MD simulations should be able to resolve questions related to local $\mathrm{H}$-bond kinetics and mechanisms unambiguously. However, even given abundant simulation data, understanding $\mathrm{H}$-bond kinetics and mechanisms is challenging for the human analyst since the relative conformation of only two water molecules is described by a six-dimensional space (one separation coordinate, two relative angular coordinates, and three relative orientational coordinates). Consequently, the state space of three water molecules, the minimal system where collective H-bond switching can be studied, is 12-dimensional, which makes the direct observation of $\mathrm{H}$-bond dynamics in simulation trajectories prohibitively difficult. In order to interpret such high-dimensional data, systematic dimensionality reduction onto a reduced space has been proven to be a useful approach. ${ }^{37,38}$

In this paper, we demonstrate that techniques used for constructing Markov state models (MSMs) ${ }^{39-41}$ provide a suitable analysis framework to study water dynamics in the complete continuum conformational space (spanned by positional and orientational angles and relative separations) and to classify the competing dynamic normal modes of $\mathrm{H}$-bond rearrangements in an unbiased and complete fashion.

MSMs have been proven useful to describe the slow dynamics in systems with many degrees of freedom, for example, protein folding, ${ }^{42,43}$ protein ligand-binding, ${ }^{44-46}$ and protein conformation changes. ${ }^{47,48}$ There are only few studies in which MSMs have been developed that account for solvent degrees of freedom, ${ }^{49,50}$ mostly because the solvent diffusion makes it difficult to define the proper subspace of relevant solvent degrees of freedom. As of yet, MSMs have not been used to analyze the coupling between orientational and translational water degrees of freedom, although this coupling has been demonstrated to be crucial for liquid water. ${ }^{51}$ Here we employ MSMs to analyze and understand the complex multi-dimensional solvent dynamics without prior definition of what a hydrogen bond is. Instead, the metastable hydrogen-bonded water structures come out as eigenstates of the transition matrix extracted from the MD data.

Based on classical simulation trajectories of liquid pure water at ambient temperature $T=300 \mathrm{~K}$, we in the first part construct a MSM in the full 12-dimensional configurational space of three water molecules and show that a few slow reconfiguration processes in the ps range can be distinguished and analyzed in terms of the cooperative rearrangements of their H-bond patterns. These slow processes are followed by a quasi-continuum of faster processes with characteristic times below 2 ps, which is consistent with the experimental finding that water relaxation processes span a wide range of time scales. In the second part, we analyze an individual H-bond switching event, defined as the process where a given central water molecule acts as a donor and the accepting $\mathrm{H}$-bond partner switches from one water molecule to a different one. This H-bond switching event has been identified as a central element of $\mathrm{H}$-bond dynamics in liquid water, and consequently, it has been amply studied. ${ }^{14,18,19}$ By projection on H-bond states, we perform a full MSM analysis of this H-bond switch scenario and provide the complete transition pathway network and analyze the competing transition probabilities. Here we basically confirm that H-bond switching mostly proceeds without intermediates, ${ }^{14,18,19}$ but also show the strength of our approach by demonstrating the existence of a significant fraction of $\mathrm{H}$-bond switching events that involve intermediate states with alternative or weak H-bonds.

\section{RESULTS AND DISCUSSION}

\section{A. Dynamics of $\mathrm{H}$-bond rearrangements in three-water clusters}

We base our analysis on trajectories of three water molecules, denoted by $\mathrm{O}^{*}, \mathrm{O}^{\mathrm{a}}$, and $\mathrm{O}^{\mathrm{b}}$, which are embedded in the liquid water environment. In order to select the set of considered water molecules, we first determine the radial pair distribution function $g_{\mathrm{OO}}(R)$ for the separation between two water oxygens, from which we derive the free energy

$$
F(R)=-k_{\mathrm{B}} T \ln \left[g_{\mathrm{OO}}(R)\right]-k_{\mathrm{B}} T \ln \left(R^{2}\right),
$$

depicted in Figs. 1(a) and 1(b). The yellow and green domains correspond to the first and second hydration shells that are separated by a free-energy barrier of about $1 k_{\mathrm{B}} T$. In previous work, we had determined the position-dependent relative diffusivity profile $D(R)$ between two water molecules using the mean first-passage time (MFPT) method, which maps the relative water dynamics onto the generalized Fokker-Planck equation. ${ }^{52}$ Our previous results in Fig. 1(c) (red solid line) show that for small separations around $R=0.3 \mathrm{~nm}$, the diffusivity $D(R)$ is six times smaller than the bulk value. Previously we had associated this decrease of $D(R)$ with the presence of orthogonal degrees of freedom that we speculated to be related to water orientational dynamics. In Fig. 1(c), we also show the diffusivity profile from our MSM model (blue circles), which results from a projection of the Markov state dynamics onto the separation coordinate $R$ (see Sec. III of the supplementary material for details). The good agreement between the results from these two very different methods is a crucial consistency check and, in particular, demonstrates that the slowed down translational water dynamics in the first hydration shell is indeed caused by the collective motion of water molecules 


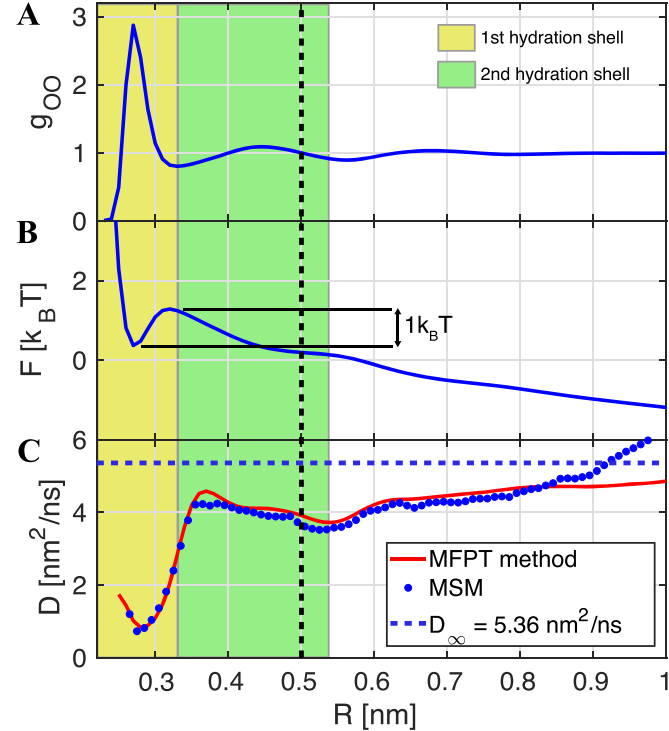

FIG. 1. (a) Radial pair distribution function $g_{\mathrm{OO}}(R)$ between two water molecules. The separation $R=0.5 \mathrm{~nm}$ (denoted by a vertical broken line) has been chosen as a cutoff for the selection of trajectories for the threewater MSM. (b) Free energy landscape $F(R)$. (c) Radial diffusivity profile $D(R)$ between two water molecules. The diffusivity profile calculated from the MSM is consistent with the profile previously estimated from the mean first-passage time (MFPT) method. $D_{\infty}$ (horizontal blue dashed line) denotes the radial diffusion constant of two diffusing waters.

that couples translational and orientational degrees of freedom (and not an artifact of our previous projection on a 1D Fokker-Planck equation).

We start recording the 12-dimensional trajectories for the three-water MSM as soon as the radial separation between $\mathrm{O}^{*}$ and $\mathrm{O}^{\mathrm{a}}, R_{\mathrm{O}^{*} \mathrm{O}^{\mathrm{a}}}$, and between $\mathrm{O}^{*}$ and $\mathrm{O}^{\mathrm{b}}, R_{\mathrm{O}^{*} \mathrm{O}^{\mathrm{b}}}$, are both $<0.5 \mathrm{~nm}$. We stop recording when one of the two waters leaves the cut-off radius of $0.5 \mathrm{~nm}$; see Fig. 2 for a sample trajectory and graphical definition of the separation coordinates $R_{\mathrm{O}} \mathrm{O}^{\mathrm{a}}$ and $R_{\mathrm{O}^{*} \mathrm{O}^{\mathrm{b}}}$. We capture 320000 trajectories with a total length of $1700 \mathrm{~ns}$ and use the $\mathrm{k}$-means++ algorithm ${ }^{53}$ with 500 cluster states in the 12-dimensional state space. We varied the number of states (see the supplementary material, Sec. IV), but found no advantage in using more than 500 clusters. The transition
$\mathbf{A}$
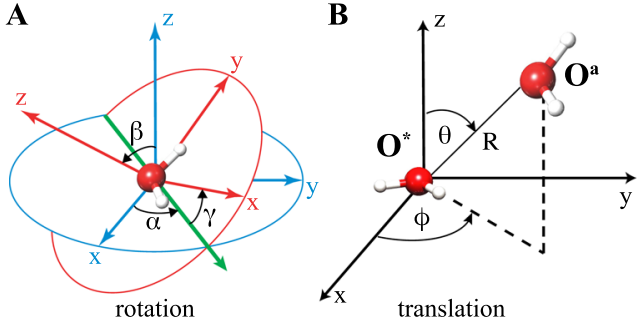

C

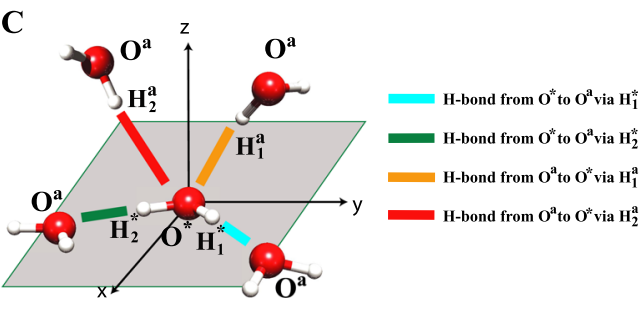

FIG. 3. (a) Euler angles are used for the rotational degrees of freedom and (b) spherical coordinates are used for the translational degrees of freedom. The reference water $\mathrm{O}^{*}$ is placed in the coordinate center such that its two hydrogens are located in the $x-y$ plane and the dipole vector points along the $x$-axis. (c) Sketch of different H-bond configurations between two waters. In the cyan configuration, $\mathrm{O}^{*}$ acts as a donor via $\mathrm{H}_{1}^{*}$ to $\mathrm{O}^{\mathrm{a}}$; in the green configuration, it donates via $\mathrm{H}_{2}^{*}$ to $\mathrm{O}^{\mathrm{a}}$. In the orange configuration, $\mathrm{O}^{*}$ accepts an $\mathrm{H}$-bond via $\mathrm{H}_{1}^{a}$, whereas in the red configuration, it accepts via $\mathrm{H}_{2}^{a}$.

probability matrix $\mathbf{T}(\tau)$ is estimated for a range of lag times $\tau$, and the implied time scales $t_{i}^{*}$ are derived from the eigenvalues $\lambda_{i}$ of $\mathbf{T}(\tau)$ (see Sec. IV).

For our MSM analysis, we fix the reference water molecule $\mathrm{O}^{*}$ in the coordinate center oriented such that its two hydrogens lie in the $x-y$ plane and the dipole vector points in the $x$ direction. The configuration of a second water molecule is then described by three coordinates for the rotation $(\alpha, \beta$, $\gamma$ ) and three coordinates for the translation in spherical coordinates $(R, \phi, \theta)$; see Figs. 3(a) and 3(b). Consequently, for three water molecules, the complete state space spanned by the relative coordinates is 12-dimensional.

In Fig. 4(a), we plot the five largest eigenvalues $\lambda_{i}$, and in Fig. 4(b), we plot the corresponding implied time scales $t_{i}^{*}$ as a function of the lag time $\tau$ (see Sec. IV for details). At lag times below $\tau<3$ ps, the eigenvalues exhibit a fast
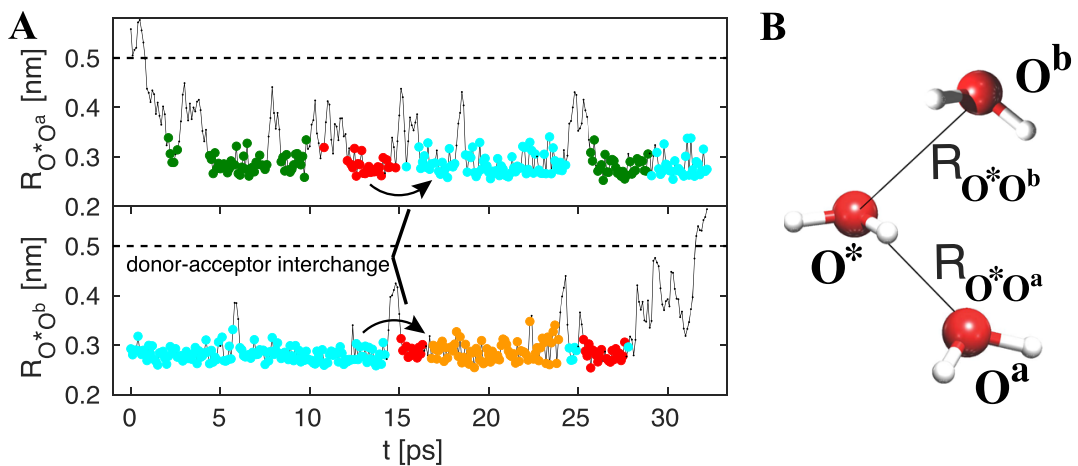

FIG. 2. (a) Trajectories of the radial distances $R_{\mathrm{O} * \mathrm{O}^{\mathrm{a}}}$ and $R_{\mathrm{O} * \mathrm{O}}$ between the oxygen of the central water and the oxygens of two neighboring water molecules. Trajectories are used for constructing the MSM when both distances are smaller than 0.5 nm, and this cutoff is denoted by broken horizontal lines. Different colors mark different $\mathrm{H}$-bonds according to the color coding in Fig. 3(c) and visualize H-bond rearrangements. For example, around $t=15$ ps, the $\mathrm{O}^{\mathrm{a}}$ water molecule changes from being a donor (red) to being an acceptor (cyan), while $\mathrm{O}^{\mathrm{b}}$ changes from an acceptor (cyan) to a donor (red). (b) Definition of the radial distances $R_{\mathrm{O}} * \mathrm{O}^{\mathrm{a}}$ and $R_{\mathrm{O} * \mathrm{O}^{\mathrm{b}}}$. 

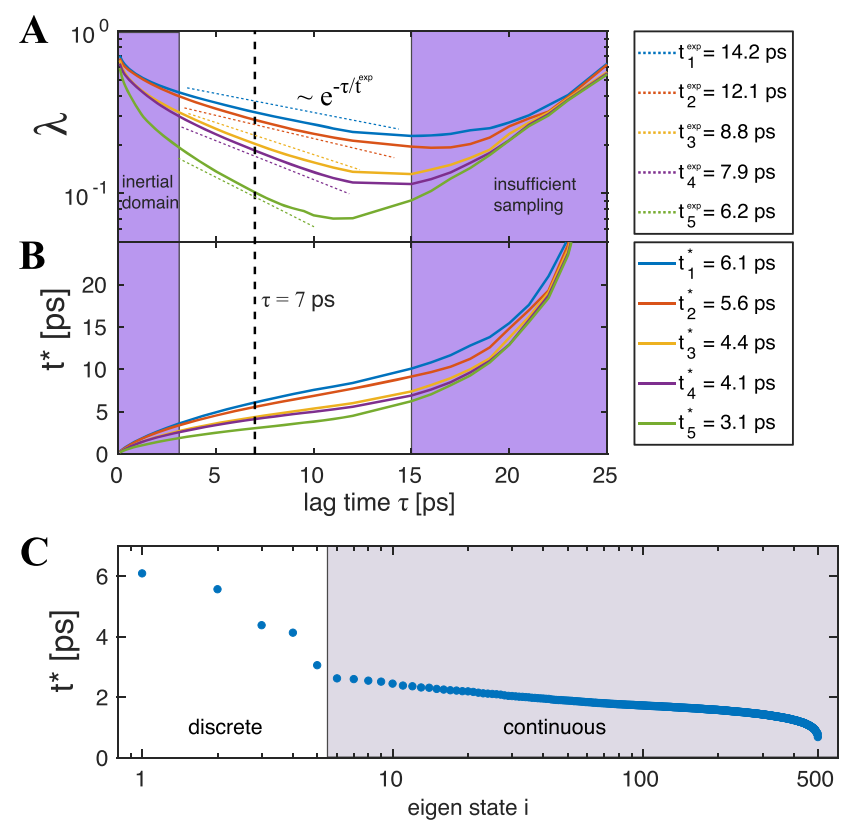

FIG. 4. (a) The first five eigenvalues $\lambda_{i}$ as a function of lag time $\tau$ for the three-water MSM. The dotted lines indicate local exponential fits leading to decay times $t_{i}^{\exp }$, given in the right panel. (b) Plot of the five slowest implied time scales $t_{i}^{*}$ versus the lag time $\tau$ for the three-water MSM. The right panel lists the implied time scales for fixed lag time $\tau=7$ ps. (c) Plot of all implied time scales for fixed lag time $\tau=7 \mathrm{ps}$. The five slowest time scales are separated from a continuum of faster processes.

decay and change of slope in the logarithmic plot, indicating non-Markovian dynamics due to inertial effects. For $\tau>20$ ps, the eigenvalues in Fig. 4(a) merge and increase, and the implied time scales in Fig. 4(b) diverge, which can be traced back to insufficient sampling (see Sec. V of the supplementary material, for a discussion and Ref. 39 for more details). In the range from 3 to $10 \mathrm{ps}$, the eigenvalues decay approximately exponentially, which indicates Markovian dynamics in this intermediate time range. The exponential decay times $t_{i}^{\text {exp }}$ are extracted by fitting exponential functions to the data, as indicated by broken straight lines in Fig. 4(a). Due to the fast inertial decay of the eigenvalues at short times, the implied time scales $t_{i}^{*}$ in Fig. 4(b), calculated according to Eq. (8), differ significantly from the exponential decay times $t_{i}^{\text {exp }}$ and also depend weakly on the lag time $\tau$. While the decay time $t_{i}^{\exp }$ describes the local relaxational decay, the implied time scale $t_{i}^{*}$ furnishes a description of the entire relaxation dynamics, which explains why $t_{i}^{*}<t_{i}^{\exp }$. However, the physical interpretation of the dynamical water arrangement processes, as extracted from the MSM eigenvectors, does not depend on the lag time, as discussed in the supplementary material, Sec. VI, which means that our MSM analysis can be used in order to investigate collective water dynamics at the picosecond time scale. In Fig. 4(c), we plot the implied time scales of all MSM eigenstates for a fixed lag time of $\tau=7 \mathrm{ps}$, and it is seen that the five slowest processes are clearly separated from a quasi-continuum band of faster relaxation processes.

In order to understand the physical meaning of the processes described by different MSM eigenstates, we project the 500 components of the eigenvectors onto the underlying 12-dimensional conformational space of the three water molecules, and for this, we choose a lag time of $\tau=7 \mathrm{ps}$. In the supplementary material, Sec. VI, we demonstrate that the interpretation of the resulting processes is not changed when the lag time is changed to $\tau=3$ ps or to $\tau=15$ ps. For clarity, we only show the projection onto the azimuthal angle $\phi$ for $\mathrm{O}^{\mathrm{a}}$ and $\mathrm{O}^{\mathrm{b}}$. The stationary distribution $\pi$, depicted in Fig. 5(a), shows pronounced clustering in four bands, at which the eigenvector amplitude and thus the distribution probability is markedly enhanced. If we color the eigenvector components according to the H-bond color coding in Fig. 3(c), we indeed see that these four bands correspond to the four different $\mathrm{H}$-bonds that can form between $\mathrm{O}^{*}$ and $\mathrm{O}^{\mathrm{a}}$ and between $\mathrm{O}^{*}$ and $\mathrm{O}^{\mathrm{b}}$. We stress that our Markov state model is constructed in an unbiased fashion and is only based on the distances in the 12-dimensional configurational space, i.e., the presence of $\mathrm{H}$-bonds is not presumed when constructing the model. On the other hand, it transpires that the resulting state clusters can a posteriori be straightforwardly associated with H-bonds.

The slowest process with a time scale $t_{1}^{*}=6.1 \mathrm{ps}$ is depicted in Fig. 5(b). The eigenvector contains negative components, which describe a loss of probability, and positive components, which describe a gain of probability, and thus corresponds to a transition between two states in configurational space. Interpretation of this transition is possible by projection on H-bonds, visualized by our color coding: For $\mathrm{O}^{\mathrm{a}}$, the green and cyan $\mathrm{H}$-bond configurations (in which $\mathrm{O}^{*}$ donates to $\mathrm{O}^{\mathrm{a}}$ ) are predominantly negative, whereas the red and orange $\mathrm{H}$-bond configurations (in which $\mathrm{O}^{*}$ accepts from $\mathrm{O}^{\mathrm{a}}$ ) are positive. For $\mathrm{O}^{\mathrm{b}}$, we observe the opposite, the red and orange configurations are negative and the green and cyan configurations are positive. This means that the slowest time scale is caused by a transition where in the initial configuration, $\mathrm{O}^{\mathrm{a}}$ accepts an $\mathrm{H}$-bond from $\mathrm{O}^{*}$ and $\mathrm{O}^{\mathrm{b}}$ donates an $\mathrm{H}$-bond to $\mathrm{O}^{*}$, while in the final configuration, $\mathrm{O}^{\mathrm{b}}$ accepts an $\mathrm{H}$-bond from $\mathrm{O}^{*}$ and $\mathrm{O}^{\mathrm{a}}$ donates an $\mathrm{H}$-bond to $\mathrm{O}^{*}$, as illustrated at the bottom of Fig. 5(b) (note that the reverse process is of course described by the same eigenvector with a negative amplitude). This process thus describes the interchange of the donor and acceptor position of $\mathrm{O}^{\mathrm{a}}$ and $\mathrm{O}^{\mathrm{b}}$ and is named as the donor-acceptor interchange. It is achieved by a concerted rotation of all three water molecules and occurs in the trajectory in Fig. 2 around $t=15 \mathrm{ps}$, where water $\mathrm{O}^{\mathrm{a}}$ changes from red to cyan (donating $\rightarrow$ accepting), whereas $\mathrm{O}^{\mathrm{b}}$ changes from cyan to red (accepting $\rightarrow$ donating). Note that this process presumably involves other water molecules as well, which however will not modify the extracted time scale and the eigenvector components shown in Fig. 5(b). This robustness of our three-water MSM with respect to changing the number of involved water molecules is demonstrated by the fact that even a more restricted two-water MSM yields the same time scales, as shown in the supplementary material, Sec. VII.

The second slowest process with a time scale of $t_{2}^{*}=5.6$ ps, depicted in Fig. 5(c), describes an acceptoracceptor interchange. Here the dominant negative part of the $\mathrm{O}^{\mathrm{a}}$ eigenvector in cyan describes an initial state where the central water $\mathrm{O}^{*}$ donates an $\mathrm{H}$-bond, whereas $\mathrm{O}^{\mathrm{b}}$ is initially described by the green configuration where the central water 


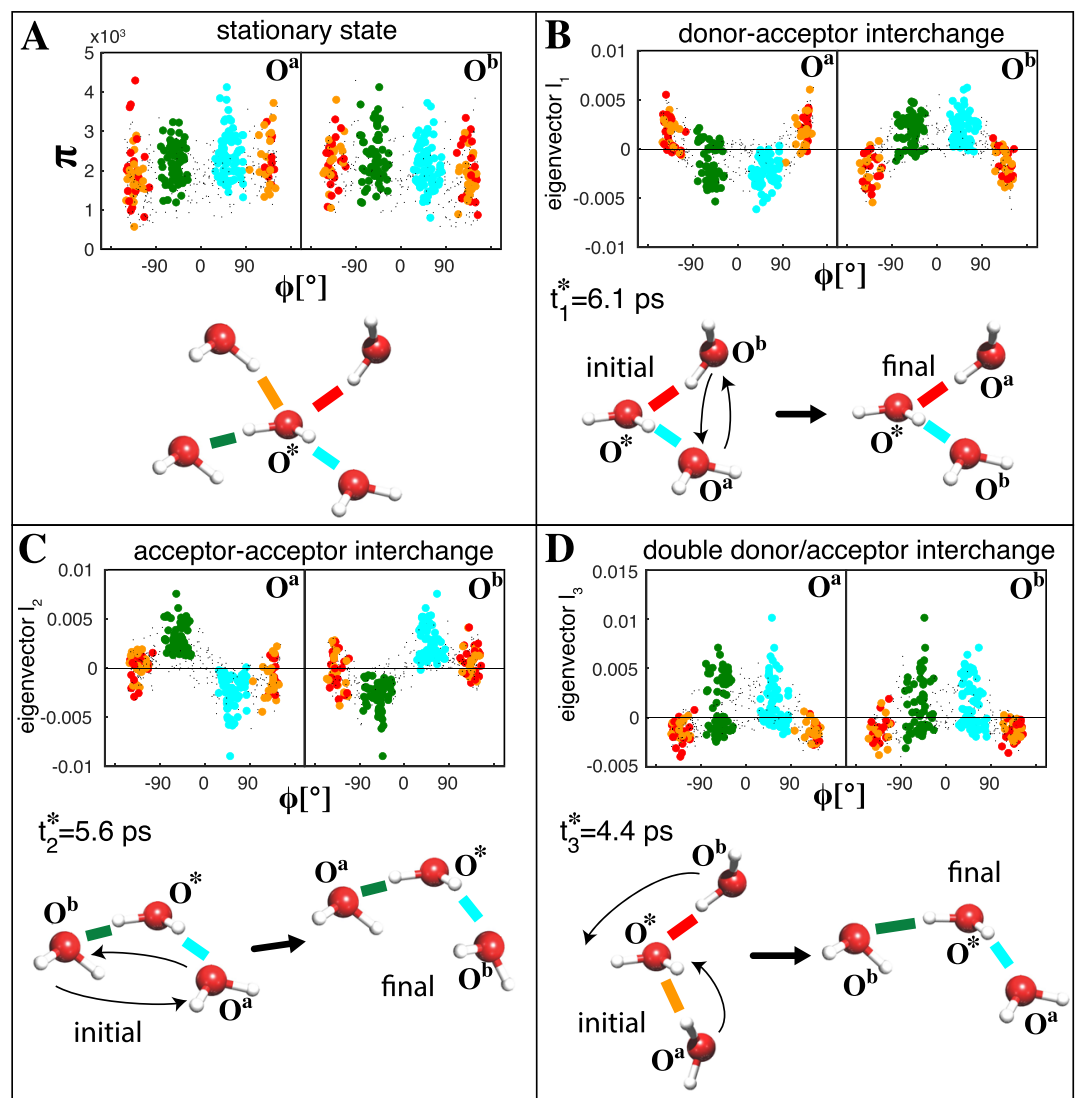

FIG. 5. Equilibrium state and the three slowest hydrogen-bond interchange processes of the three-water MSM. We show the projection of the eigenvector onto the azimuthal angle $\phi$ for the water pairs $\mathrm{O}^{*}-\mathrm{O}^{\mathrm{a}}$ and $\mathrm{O}^{*}-\mathrm{O}^{\mathrm{b}}$. Colored eigenvector components denote H-bonds according to the definition shown in (a). (a) The equilibrium state consists of a combination of $\mathrm{H}$-bonds between $\mathrm{O}^{*}$ and the waters $\mathrm{O}^{\mathrm{a}}$ and $\mathrm{O}^{\mathrm{b}}$. (b) The eigenvector associated with the slowest time scale $t_{1}^{*}=6.1$ ps describes a donor-acceptor interchange, where $\mathrm{O}^{\mathrm{a}}$ and $\mathrm{O}^{\mathrm{b}}$ interchange their roles as an acceptor and a donor. This process is achieved by a collective rotation of all three waters $\mathrm{O}^{*}, \mathrm{O}^{\mathrm{a}}$, and $\mathrm{O}^{\mathrm{b}}$ in the lab frame. Note that for clarity, we show water configurations in the coordinate system centered on the central water molecule $\mathrm{O}^{*}$. (c) Acceptor-acceptor interchange with a time scale of $t_{2}^{*}=5.6 \mathrm{ps}$. Here $\mathrm{O}^{*}$ donates $\mathrm{H}$-bonds to both waters $\mathrm{O}^{\mathrm{a}}$ and $\mathrm{O}^{\mathrm{b}}$ via its two $\mathrm{H}$-atoms in the initial and final states. This process is achieved by a single rotation of the central water molecule $\mathrm{O}^{*}$. (d) Double donor/acceptor interchange with a time scale of $t_{3}^{*}=4.4 \mathrm{ps}$. In the initial state, $\mathrm{O}^{*}$ accepts two $\mathrm{H}$-bonds from $\mathrm{O}^{\mathrm{a}}$ and $\mathrm{O}^{\mathrm{b}}$, and in the final state, $\mathrm{O}^{\mathrm{a}}$ and $\mathrm{O}^{\mathrm{b}}$ accept $\mathrm{H}$-bonds from $\mathrm{O}^{*}$.

$\mathrm{O}^{*}$ also donates an $\mathrm{H}$-bond. In the final configuration, $\mathrm{O}^{\mathrm{a}}$ and $\mathrm{O}^{\mathrm{b}}$ have interchanged positions. Thus, this process is an acceptor-acceptor interchange, which can be achieved by a single rotation of the central water $\mathrm{O}^{*}$ by $180^{\circ}$ around its dipole axis. In the third slowest process with $t_{3}^{*}=4.4 \mathrm{ps}$ in Fig. 5(d), both waters $\mathrm{O}^{\mathrm{a}}$ and $\mathrm{O}^{\mathrm{b}}$ initially donate $\mathrm{H}$-bonds to $\mathrm{O}^{*}$ (negative orange and red eigenvector components), whereas in the final state, both waters accept $\mathrm{H}$-bonds from $\mathrm{O}^{*}$ (predominantly positive blue and cyan eigenvector components). This transition can be achieved by a concerted rotation of all three water molecules and corresponds to a double donor/acceptor interchange. The faster processes become more and more difficult to interpret since they do not allow for a clear-cut analysis in terms of H-bond patterns.

We conclude that our three-water MSM clearly reveals the slowest H-bond rearrangement patterns in a water trimer that is embedded in liquid water. The resulting slowest time scales $t_{i}^{*}$ are in the range of 4-6 ps and thus considerably slower than the typical librational time scale of $t^{\text {libr }} \sim 200 \mathrm{fs}^{22,54,55}$ or the reorientational time scale of $t^{\text {reorient }} \sim 2.5 \mathrm{ps}$ it takes an $\mathrm{H}$-bond donating water to switch from one to a different acceptor. ${ }^{18-20,22,56}$ This is not difficult to rationalize since the three processes presented in Fig. 5 involve the breaking and reformation of at least two H-bonds. For the second slowest process with $t_{2}^{*}=5.6 \mathrm{ps}$ in Fig. 5(c), the H-bond arrangement can proceed via the rotation of a single water molecule; for the slowest and third slowest processes in Figs. 5(b) and $5(d)$, the arrangement involves the concerted reconfiguration of the entire water trimer. So there is no simple correlation between the relaxation time of a process and the number of water molecules that reorient during the process, which is an interesting finding.

The comparison of the relaxation times we extract from our MSM analysis with experimental results is not straightforward. On the one hand, different experiments measure different relaxation times and it is not clear whether they actually measure the same processes. Even the linear absorption spectrum of pure liquid water is complex and the interpretation of the various spectroscopic signatures between the dielectric main peak at $20 \mathrm{GHz}$ and the infra-red $\mathrm{OH}$ bend and $\mathrm{OH}$ stretch bands (around 50 and $100 \mathrm{THz}$ ) is still a subject of intense discussion. ${ }^{57-59}$ On the other hand, not all processes our MSM analysis reveals are spectroscopically active, for example, the second slowest process corresponds to the rotation of a single water around its dipolar axis and does not involve a polarization change; it will therefore not be seen in absorption experiments. 
Also, it should be kept in mind that our MSM analysis captures barrier-crossings between metastable states; this means that normal modes, i.e., vibrations around a metastable structure, which are ordinarily used to model spectroscopic bands, are not included. Even with these restrictions, a number of conclusions with respect to experimental spectra can be drawn: In photon-echo experiments, ${ }^{33}$ it was found that a comparatively slow time scale in the 5-15 ps range exists that was suggested to be related to H-bond rearrangements. The exponential relaxation time scale $t_{1}^{\exp }$ of the slowest eigenstate extracted from a local fit of the eigenvalue in Fig. 4(a) is of the order of $t_{1}^{\text {exp }}=14$ ps while the slowest implied time scale is $t_{1}^{*}=6.1 \mathrm{ps}$; both are quite close to the experimental value, but it is not clear whether a photon echo experiment is dominated by the local decay time $t_{i}^{\text {exp }}$ or by the implied time scale $t_{i}^{*}$. The times $t_{1}^{*}=6.1 \mathrm{ps}$ and $t_{1}^{\exp }=14 \mathrm{ps}$ correspond to frequencies of $f=1 /(2 \pi \times 6.1) \mathrm{Hz}=26.1 \mathrm{Ghz}$ and $f=11.4 \mathrm{Ghz}$, not so different from the main dielectric relaxation peak of SPC/E water at $f=15 \mathrm{GHz}$ found in simulations of SPC/E water. ${ }^{7}$ So in essence, the relaxation time scales we extract with our analysis agree with the experimental time scales at which water is suggested to change its local hydrogen bonding structure. Our finding of an entire set of distinct slow dynamic collective water modes in the picosecond range might explain why experimentally measured time scales differ substantially, different experiments might indeed pick up different elementary water restructuring processes.

It is interesting that the single water rotation mode in Fig. 5(c) has almost the same time scale as the other two concerted rotation transitions in Figs. 5(b) and 5(d) and that all three modes describe very different structural rearrangements of the H-bonding pattern within the water trimer. This vividly demonstrates why the interpretation of the microscopic water dynamics is so complex, even when simulation trajectories with full configurational information are available. It would be hard to extract the dynamical modes shown in Fig. 5 from the simulation trajectories without the MSM analysis. In the supplementary material, Sec. VII, we show that a restricted MSM for a water dimer gives quite similar slow time scales but obviously does not allow an interpretation of the kinetic processes in terms of collective $\mathrm{H}$-bond reconfigurations. This shows that the resulting time scales of the water MSM are robust with respect to the number of water molecules the MSM is based upon.

\section{B. Transition-path network for H-bond acceptor switching}

In Sec. II A, we analyzed the slowest elementary processes that occur in a three-water MSM and showed that they correspond to the simultaneous breaking and reforming of at least two H-bonds. In this section, we pursue a somewhat simpler question, namely, the switching of a single H-bond from one accepting water molecule to a different accepting water molecule. Note that this single $\mathrm{H}$-bond switching process is contained in the more complete three-water scenario discussed in Sec. II A. In fact, the mechanism and the transition pathway of this single $\mathrm{H}$-bond switching event have been challenging topics of research for decades. ${ }^{17}$ In Ref. 16, it has been shown via transition path sampling that in roughly half of the cases when an $\mathrm{H}$-bond is broken, a new H-bond forms right after. In Refs. 18 and 19, it has been shown that this H-bond switch is dominated by an abrupt angular rotation of the central water molecule that acts as a donor. Here we will bring these two findings in harmony with each other and, in particular, will analyze

A

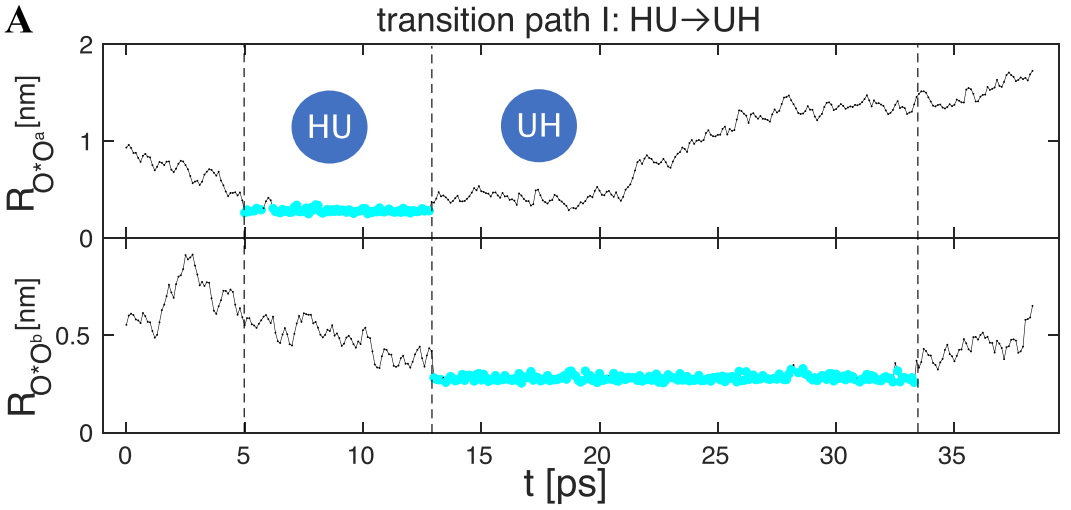

B

transition path $\mathrm{V}: \mathrm{HU} \rightarrow \mathrm{aH} \rightarrow \mathrm{UH}$

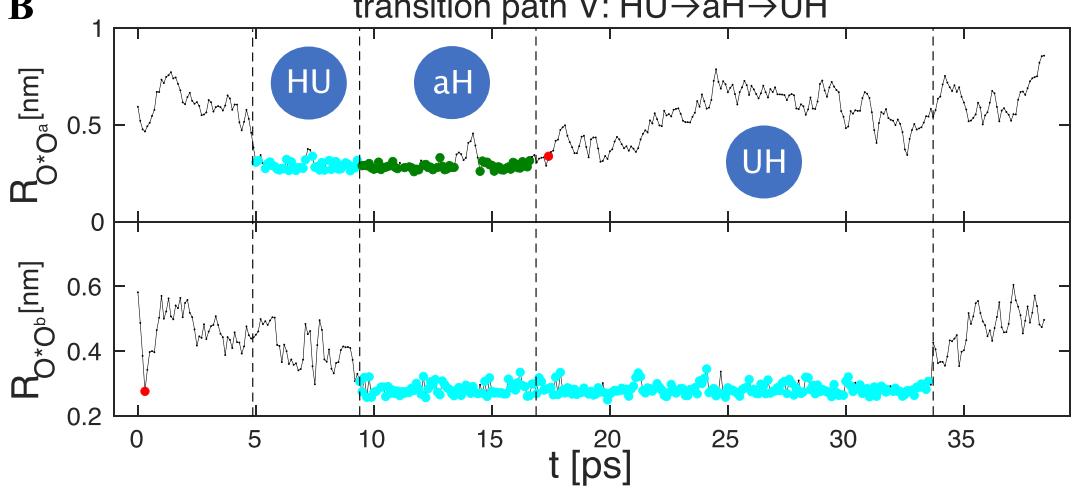

FIG. 6. Sample trajectories of H-bond switching events in terms of the radial distances $R_{\mathrm{O}^{*} \mathrm{O}^{\mathrm{a}}}$ and $R_{\mathrm{O} * \mathrm{O}^{\mathrm{b}}}$ between three water molecules. (a) At $t=5 \mathrm{ps}$, an $\mathrm{H}$-bond (cyan color) between $\mathrm{O}^{*}$ and $\mathrm{O}^{\mathrm{a}}$ is formed for the first time. At $t=13 \mathrm{ps,}$ this $\mathrm{H}$-bond between $\mathrm{O}^{*}$ and $\mathrm{O}^{\mathrm{a}}$ is broken and $\mathrm{O}^{*}$ immediately switches to $\mathrm{O}^{\mathrm{b}}$. After this, $\mathrm{O}^{\mathrm{a}}$ diffuses away from $\mathrm{O}^{*}$ while $\mathrm{O}^{*}$ and $\mathrm{O}^{\mathrm{b}}$ stay $\mathrm{H}$-bonded until the H-bond is finally broken at $t=34 \mathrm{ps}$. This process is an example for the direct transition path type I; see Fig. 7(c). (b) At $t=5 \mathrm{ps}$, an $\mathrm{H}$-bond (cyan color) between $\mathrm{O}^{*}$ and $\mathrm{O}^{\mathrm{a}}$ is formed for the first time. At $t=9 \mathrm{ps}$, this H-bond switches to $\mathrm{O}^{\mathrm{b}}$ and $\mathrm{O}^{\mathrm{a}}$ forms an alternative $\mathrm{H}$ bond with $\mathrm{O}^{*}$ where it accepts a different hydrogen from $\mathrm{O}^{*}$ (denoted by green color) until it diffuses away at $t=16.5$ ps. This process is an example of transition path type V; see Fig. 7(c). The color notation of H-bonds is defined in Fig. 3. 
the complete transition network that describes the switching of a single $\mathrm{H}-$ bond acceptor.

In order to describe the switching event of a single $\mathrm{H}$ bond acceptor by a MSM, we modify the selection rule for trajectories used in Sec. II A and project onto a much more restricted set of states. We now consider trajectories where $\mathrm{O}^{*}$ is $\mathrm{H}$-bonded to a second water $\mathrm{O}^{\mathrm{a}}$ via its hydrogen atom $\mathrm{H}_{1}^{*}$ initially and switches to a third water $\mathrm{O}^{\mathrm{b}}$ to which it forms an $\mathrm{H}$ bond via the same hydrogen $\mathrm{H}_{1}^{*}$. We start recording trajectories as soon as $\mathrm{O}^{*}$ and $\mathrm{O}^{\mathrm{a}}$ become $\mathrm{H}$-bonded under the condition that no $\mathrm{H}$-bond between $\mathrm{O}^{*}$ and $\mathrm{O}^{\mathrm{b}}$ exists and stop when the $\mathrm{H}$-bond between $\mathrm{O}^{*}$ and $\mathrm{O}^{\mathrm{b}}$ is broken finally; see Figs. 6(a) and 6(b) for two typical trajectories.

We collect a total of 199197 H-bond switching events and define four basis states which describe the $\mathrm{H}$-bond configuration between two water molecules. In the H-bonded state, called " $\mathrm{H}$," the central water molecule $\mathrm{O}^{*}$ forms an $\mathrm{H}$-bond with water molecule $\mathrm{O}^{\mathrm{x}}$ by donating its hydrogen $H_{1}^{*}$, where the $\mathrm{O}^{\mathrm{x}}$ stands for water $\mathrm{O}^{\mathrm{a}}$ or $\mathrm{O}^{\mathrm{b}}$. In the unbound state, called " $\mathrm{U}$," there is no $\mathrm{H}$-bond between $\mathrm{O}^{*}$ and $\mathrm{O}^{\mathrm{x}}$ and the radial distance between the waters is $R_{\mathrm{O}^{*} \mathrm{O}^{\mathrm{x}}}>0.35 \mathrm{~nm}$, which exceeds the threshold separation for an H-bond. In the weakly bounded state, called "w," there is no H-bond because the angular criterion is not fulfilled, but the distance between the two waters is $R_{\mathrm{O}^{*} \mathrm{O}^{\mathrm{x}}}<0.35 \mathrm{~nm}$. In the alternative H-bond state, called "a," the water molecule $\mathrm{O}^{*}$ either accepts an $\mathrm{H}$-bond from $\mathrm{O}^{\mathrm{x}}$ or it forms an $\mathrm{H}$-bond with $\mathrm{O}^{\mathrm{x}}$ by donating its other hydrogen $H_{2}^{*}$ to $\mathrm{O}^{\mathrm{x}}$; see Fig. 7(a) for an illustration of these four states. The combinations of these four basis states yield 4 $\times 4=16 \mathrm{MSM}$ cluster states for three water molecules $\mathrm{O}^{*}, \mathrm{O}^{\mathrm{a}}$, and $\mathrm{O}^{\mathrm{b}}$.

In the reactant state, $\mathrm{O}^{*}$ and $\mathrm{O}^{\mathrm{a}}$ are in state $\mathrm{H}$, whereas $\mathrm{O}^{*}$ and $\mathrm{O}^{\mathrm{b}}$ are in state $\mathrm{U}$, which we denote as $\mathrm{HU}$ (i.e., the first letter denotes the bonding state between $\mathrm{O}^{*}$ and $\mathrm{O}^{\mathrm{a}}$ and the second letter denotes the bonding state between $\mathrm{O}^{*}$ and $\mathrm{O}^{\mathrm{b}}$ ). The product state is defined as $\mathrm{UH}$ where water molecules $\mathrm{O}^{\mathrm{a}}$ and $\mathrm{O}^{\mathrm{b}}$ have exchanged their roles; see Fig. 7(b). The validity of the defined MSM for a lag time of $\tau=1 \mathrm{ps}$ is discussed in the supplementary material, Sec. VIII. The transition rate turns out to be $k_{\mathrm{HU} \rightarrow \mathrm{UH}}=0.24 \mathrm{ps}^{-1}$ (see Sec. IV and the supplementary material, Sec. II, for details), which yields a reaction time of

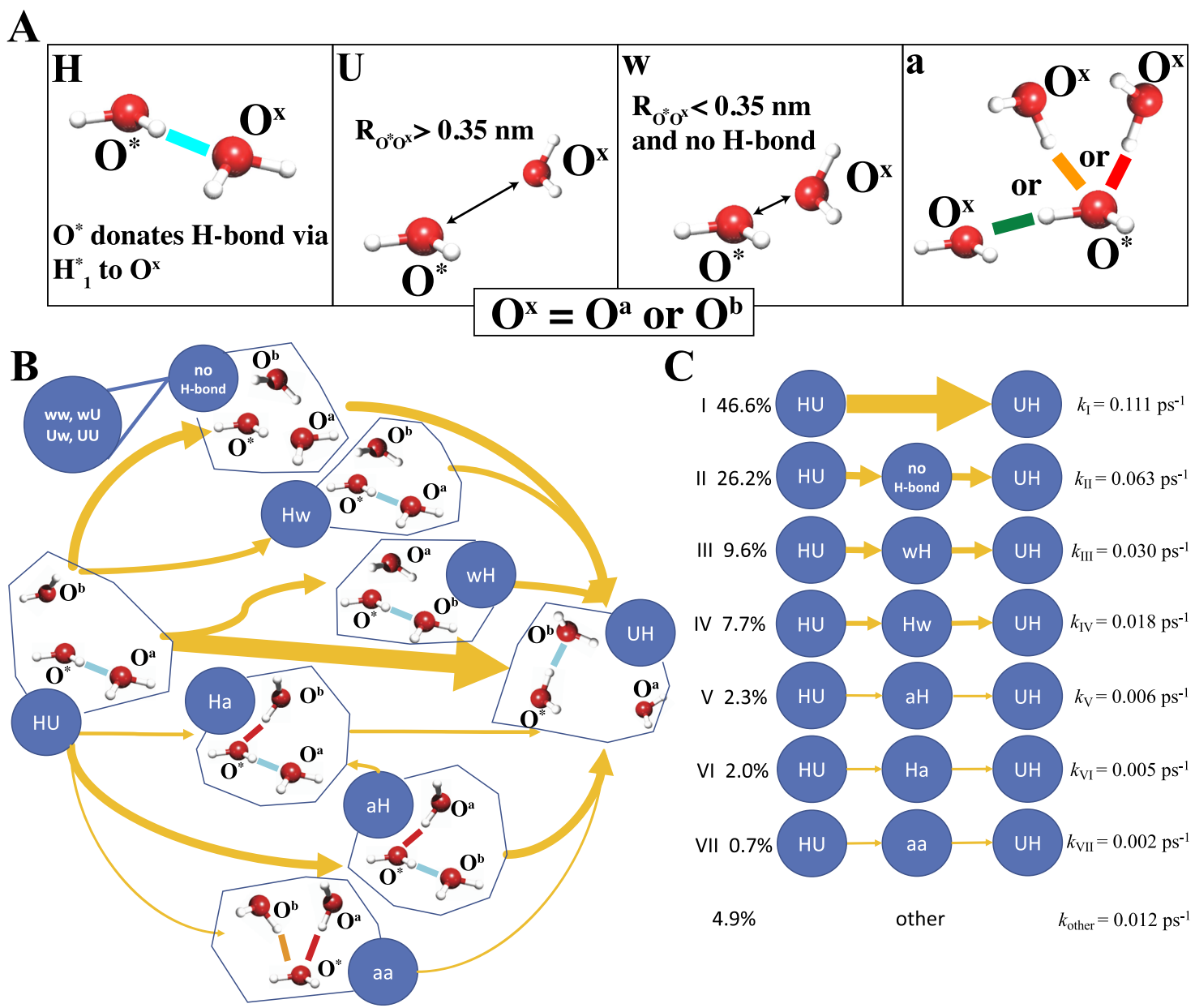

FIG. 7. (a) The four basis states describing the bonding state between the central water molecule $\mathrm{O}^{*}$ and a second water molecule $\mathrm{O}^{\mathrm{x}}$. We distinguish state "H," where $\mathrm{O}^{*}$ is via $H_{1}^{*}$ hydrogen bonded to $\mathrm{O}^{\mathrm{x}}$, state "U," where no bond exists between $\mathrm{O}^{*}$ and $\mathrm{O}^{\mathrm{x}}$, the weakly bound state "w," and the alternative bound state "a." (b) Transition network for the H-bond acceptor switching, showing only the eight dominant pathways, where the thickness of arrows indicates the net-flux. In the reactant state $\mathrm{HU}, \mathrm{O}^{*}$ acts as an $\mathrm{H}$-bond donor to $\mathrm{O}^{\mathrm{a}}$ via $\mathrm{H}_{1}^{*}$, while no $\mathrm{H}$-bond with $\mathrm{O}^{\mathrm{b}}$ is present and the distance between $\mathrm{O}^{*}$ and $\mathrm{O}^{\mathrm{b}}$ is larger than 0.35

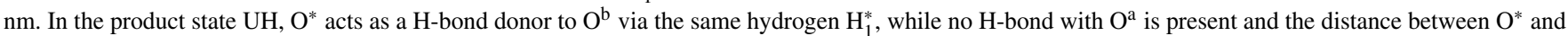
$\mathrm{O}^{\mathrm{a}}$ is larger than $0.35 \mathrm{~nm}$. (c) Transition pathways ordered by their reaction probabilities (to the left) or reaction rates (to the right). Pathways with probability lower than $0.5 \%$ are not shown. 


$$
\tau_{\mathrm{HU} \rightarrow \mathrm{UH}}=1 / k_{\mathrm{HU} \rightarrow \mathrm{UH}}=4.2 \mathrm{ps} .
$$

This reaction time is smaller than the previously published estimate of the H-bond lifetime of $\tau_{\mathrm{HB} \text {-life }}=6.5$ to $7.0 \mathrm{ps},{ }^{13,19}$ but larger than the previously calculated H-bond switching time $\tau_{\text {HB-switch }}=3.3$ ps; see Refs. 18 and 19 and the supplementary material, Sec. VIII. Note that the difference between the H-bond lifetime $\tau_{\mathrm{HB}-\text { life }}$ and the H-bond switching time $\tau_{\mathrm{HB}-\text { switch }}$ reflects the fact that after an $\mathrm{H}$-bond switch, an $\mathrm{H}-$ bond switch back to the initial H-bonded configuration can occur; see Ref. 19 for details. In fact, our reaction time $\tau_{\mathrm{HU} \rightarrow \mathrm{UH}}$ includes the diffusion of $\mathrm{O}^{\mathrm{b}}$ towards $\mathrm{O}^{*}$ and the diffusion of $\mathrm{O}^{\mathrm{a}}$ away from $\mathrm{O}^{*}$ and is therefore slightly larger than the $\mathrm{H}$-bond switching time $\tau_{\mathrm{HB} \text {-switch }}$.

The 16 states and the fluxes create a transition network, which can be decomposed into competing pathways that are characterized by different transition probabilities and reaction times; see the supplementary material, Sec. II, for the detailed derivation. We show the complete transition network in Fig. 7(b). The thickness of the arrows indicates the net flux. States which contribute with a net flux close to zero have been omitted, and as a consequence of this, the transition network in Fig. 7(b) shows only 11 of the 16 possible states defined by the MSM. As the most important result, we find that competing pathways for the H-bond switching exist. The main transition pathway I with a probability of $46.6 \%$ and a reaction rate of $k_{I}=0.111 \mathrm{ps}^{-1}$ is the direct transition path $\mathrm{HU} \rightarrow \mathrm{UH}$, where $\mathrm{O}^{*}$ switches its donating hydrogen from $\mathrm{O}^{\mathrm{a}}$ to $\mathrm{O}^{\mathrm{b}}$ without an intermediate state (on the time scale set by the lag time of $\tau=1 \mathrm{ps}$ used in this section). The transition pathways III and IV involve the weakly bound state $\mathrm{w}$, where $\mathrm{O}^{\mathrm{a}}$ or $\mathrm{O}^{\mathrm{b}}$ is in an intermediate state with a separation $R<$ $0.35 \mathrm{~nm}$. The three transition pathways I, III, and IV sum up to about two thirds of all possible transitions; they describe a direct H-bond switch between the two accepting water molecules and thus correspond to the pathway described by Laage et al. ${ }^{18,19}$ and indeed constitute the predominant pathway for H-bond switching in agreement with Ref.16.

However, the second important pathway II consists of a short-lived intermediate state which can be of the ww, wU, Uw, or UU type and has a probability of $26.2 \%$. The other group of pathways, V, VI, and VII, involves intermediate states where $\mathrm{O}^{*}$ forms an alternative $\mathrm{H}$-bond with $\mathrm{O}^{\mathrm{a}}$ or $\mathrm{O}^{\mathrm{b}}$, and they sum up to a total probability of $5.0 \%$. An example of a trajectory of type IV is shown in Fig. 6(b), where the $\mathrm{O}^{\mathrm{a}}$ water does not immediately diffuse away from $\mathrm{O}^{*}$ after breaking its initial $\mathrm{H}$-bond with $\mathrm{O}^{*}$, but rather stays $\mathrm{H}$-bonded to $\mathrm{O}^{*}$ via an alternative $\mathrm{H}$-bond. Together, all pathways where the $\mathrm{H}$-bond does not switch directly from $\mathrm{O}^{\text {a }}$ to $\mathrm{O}^{\mathrm{b}}$ make up about one third of all switches and thus are not negligible. Clearly, by extracting averaged coordinates during H-bond switches from simulation trajectories or by looking at dominant transition pathways from transition path sampling, such subdominant alternative pathways are easily overlooked. Nevertheless, such subdominant pathways might be picked up in certain experiments and therefore could play an important role for the correct interpretation of experimental data.

\section{CONCLUSIONS}

We use Markov state modeling techniques for the analysis of liquid water structural dynamics based on classical molecular dynamics simulations. In the first part, we consider water dynamics in the full 12-dimensional continuum space of a water trimer that is embedded in liquid water and identify the slowest barrier crossing processes and relate them to dynamical rearrangements of H-bond patterns. The three slowest processes consist of the breaking and reforming of at least two H-bonds and correspond to donor-acceptor, acceptor-acceptor, and double donor/acceptor interchanges. Interestingly, the second slowest process, the acceptor-acceptor interchange, corresponds to the rotation of a single water molecule, while the donor-acceptor and double donor/acceptor interchanges involve the concerted rotation of all three water molecules. This means that slow water processes consist of either collective [like the slowest and third-slowest process in Figs. 5(b) and $5(\mathrm{c})$ ] or single-water reorientation processes [like the second-slowest process in Fig. 5(c)]. In other words, collective processes are not necessarily slower than single-molecule processes.

In the second part, we formulate the MSM in a much more restricted state space that results from a prior $\mathrm{H}-$ bond projection. Using transition path analysis, we classify all possible pathways describing the H-bond switching from one accepting water molecule to a second accepting water molecule. The dominant transition pathways correspond to a direct transition to the new H-bond acceptor without a broken $\mathrm{H}$-bond as an intermediate state, which make up about $66 \%$ of all transitions and have been investigated before. ${ }^{16,18,19}$ A non-H-bonded intermediate occurs in the transition pathway in about $26 \%$ of all transitions while in $5 \%$ of all transitions, an alternative H-bond arrangement occurs in the intermediate state. We conclude that the dominant transition pathways we find for the single H-bond switch are consistent with previous results, but that MSMs allow us to draw a more complete picture of the H-bond reconfiguration dynamics.

In previous quantum calculations, the concerted breaking and reforming of H-bonds in isolated water clusters at zero temperature has been characterized. ${ }^{34-36}$ These studies suggest that $\mathrm{H}$-bond rearrangements are local and do not involve more than three water molecules. ${ }^{35}$ Nevertheless, it would be interesting to extend MSMs for bulk liquid water to tetramers or pentamers in order to check whether concerted $\mathrm{H}$-bond rearrangements that involve more than three water molecules exist in the liquid state. Our simulation trajectories are obtained from classical force field simulations and neglect the quantum nature of nuclei motion, which is an acceptable approximation at the time scale we are probing. ${ }^{60,61}$ Effects from suboptimal force fields are presumably more critical, which is an issue we leave for future studies.

As a final note, we would like to discuss whether the occurrence of abrupt angular jumps is in conflict with Debye's diffusive model. As briefly mentioned in the Introduction, the presence of an angular free energy barrier predicts a transition path time that is much shorter than the H-bond lifetime. ${ }^{25-27}$ Explicitly, the mean-first passage time to cross a harmonic barrier of angular width $L$ is for large barrier height $U$ 
given by ${ }^{27}$

$$
\tau_{\mathrm{mfp}}=\frac{\sqrt{\pi} L^{2} e^{U / k_{B} T}}{8 D\left(U / k_{B T}\right)^{3 / 2}},
$$

where $D$ is the effective angular diffusion constant along the angular reaction coordinate. By contrast, the transition path time, which means the actual duration of the path that crosses the barrier, is for large barrier height $U$ given by ${ }^{27}$

$$
\tau_{\mathrm{tp}}=\frac{\sqrt{\pi} L^{2} \ln \left(2 e^{\gamma} U / k_{B} T\right)}{8 D U / k_{B} T},
$$

where $\gamma=0.577$ is the Euler gamma constant and only logarithmically depends on the barrier height. The ratio of the transition path and the mean-first passage time is given by $^{25}$

$$
\tau_{\mathrm{tp}} / \tau_{\mathrm{mfp}}=\frac{\ln \left(2 e^{\gamma} U / k_{B} T\right)\left(U / k_{B} T\right)^{1 / 2}}{\sqrt{\pi} e^{U / k_{B} T}},
$$

which for a rescaled barrier height $U / k_{B} T=4$ takes a value of $\tau_{\mathrm{tp}} / \tau_{\mathrm{mfp}}=0.05$. This means that even for a moderate barrier height of $U / k_{B} T=4$, the angular transition is rather short and thus abrupt compared with the hydrogen bond lifetime, which does not invalidate the Debye diffusive picture but rather shows that free-energetic barrier effects must be taken into account.

\section{METHODS}

\section{A. Markov state model and transition-path network analysis}

MSMs describe the complex dynamics of an arbitrary system by a Markovian stochastic process. Relevant degrees of freedom are projected onto a finite number of discrete states, and the rates or transition probabilities between different states are described by a transition probability matrix $\mathbf{T}$. From this matrix, transition times, transition paths and their probabilities can be extracted..$^{39,40,62}$ First, the state space has to be partitioned into $N$ states. This is not trivial for a diffusive system like liquid water since the relevant state space should ideally include only the subset of water molecules that interact with each other, which obviously changes with time. From a simulated trajectory, the $N \times N$ transition probability matrix $\mathbf{T}$ is calculated. The elements $T_{i j}$ describe the conditional probability of a transition from state $i$ to $j$ within a fixed lag time $\tau$ and are estimated by $T_{i j}(\tau)=c_{i j}(\tau) / \sum_{j} c_{i j}(\tau)$, where $c_{i j}(\tau)$ is the number of transitions from state $i$ to $j$ within time $\tau$ and $\sum_{j} c_{i j}(\tau)$ is the number of transitions from $i$ to any other state within time $\tau$. The matrix is defined in such a way that the sum of every row is unity; thus, it conserves probability. We assume that the transition matrix is ergodic (any state can be reached from any other state within a finite number of steps), which yields a single eigenvector $\boldsymbol{\pi}$ with eigenvalue unity, the stationary distribution. For an MD simulation in equilibrium, the detailed balance assumption $\pi_{i} T_{i j}=\pi_{j} T_{j i}$ holds. If the $N$-dimensional vector $\mathbf{p}(t)$ describes the probability distribution at time $t$, the probability at time $t+\tau$ follows from $\mathbf{p}(t+\tau)=\mathbf{p}(t) \mathbf{T}(\tau)$. The system is Markovian if it fulfills the Chapman-Kolmogorov equation

$$
\mathbf{T}(n \tau)=\mathbf{T}^{n}(\tau) .
$$

The spectral decomposition of the transition matrix according to

$$
\begin{aligned}
\mathbf{p}(n \tau) & =\mathbf{p}(0) \mathbf{T}^{n}(\tau) \\
& =\sum_{i=1}^{N} \lambda_{i}^{n}(\tau)\left(\mathbf{p}(0) \cdot \mathbf{r}_{i}\right) \mathbf{I}_{i}^{T} \\
& =\sum_{i=1}^{N} e^{-n \tau / t_{i}^{*}}\left(\mathbf{p}(0) \cdot \mathbf{r}_{i}\right) \mathbf{l}_{i}^{T},
\end{aligned}
$$

where $\mathbf{r}_{i}$ and $\mathbf{l}_{i}^{T}$ are the $i$ th right and left eigenvectors of the transition matrix, yields characteristic time scales

$$
t_{i}^{*}=-\frac{\tau}{\ln \lambda_{i}(\tau)}
$$

that are directly related to the eigenvalues $\lambda_{i}$ and for a Markovian system independent of the lag time $\tau$. Equation (7) describes the evolution of the probability density distribution as a weighted superposition of exponentially decaying left eigenvectors. In a nutshell, the construction of a Markov state model requires (i) clustering of the phase space into $N$ states, (ii) estimating the transition matrix from data, and (iii) checking the validity of the Markov model by testing whether the implied time scales depend on the lag time $\tau$; see the supplementary material, Sec. I, for details.

From the transition matrix, transition pathways can be extracted that lead from the subspace of reactant states $A$ to the subspace of product states $B$ and pass through the subspace of intermediate states $I$. The solution of the linear system of equations

$$
-q_{i}^{B}+\sum_{k \in I} T_{i k} q_{k}^{B}=-\sum_{k \in B} T_{i k}
$$

defines the committor probability $q_{i}^{B}$, which describes the probability of reaching $B$ before returning to $A$ when being in state $i$. The flux along the intermediate states $i$ and $j$ which contribute to transitions from $A$ to $B$ is

$$
f_{i j}=\pi_{i} q_{i}^{A} T_{i j} q_{j}^{B},
$$

from which the reaction rate $k_{A B}$ follows as

$$
k_{A B}=\sum_{i \in A}^{N} f_{i j}\left(\tau \sum_{i=1}^{N} \pi_{i} q_{i}^{A}\right) .
$$

The set of states and fluxes creates a transition network, which can be decomposed into individual transition pathways with different probabilities; see the supplementary material, Sec. II, for details.

\section{B. Molecular dynamics simulations}

The MSM is based on a molecular dynamics (MD) simulation of $895 \mathrm{SPC} / \mathrm{E}$ water molecules ${ }^{63}$ in a cubic box of edge length $L=3 \mathrm{~nm}$ with periodic boundary conditions. The trajectories are generated using GROMACS ${ }^{64}$ with a Berendsen weak coupling thermostat and barostat ${ }^{65}$ with a relaxation time of $t_{\text {rel }}=1 \mathrm{ps}$ for a fixed temperature of $T=300 \mathrm{~K}$ and a pressure of $p=1$ bar. The time step of the MD simulation is $2 \mathrm{fs}$; every $20 \mathrm{fs}$, the positional coordinates of every water molecule are stored and the total simulation time is $10 \mathrm{~ns}$. The relative configuration of two water molecules is described by six coordinates: we fix the reference water molecule $\mathrm{O}^{*}$ 
in the coordinate center with its two hydrogens in the $x-y$ plane such that the dipole vector points in the $x$ direction, to describe the configuration of a second water molecule; we then have three coordinates for the rotation $(\alpha, \beta, \gamma)$, see Fig. 3(a), and three coordinates for the translation in spherical coordinates $(R, \phi, \theta)$, see Fig. 3(b). The rotation is described by Euler angles $(\alpha, \beta, \gamma)$ in the $\left(z, x^{\prime}, z^{\prime}\right)$-convention: $\mathrm{A}$ rotation around the $z$-axis by the angle $\alpha$, a rotation around the new $x$-axis $\left(x^{\prime}\right)$ by the angle $\beta$, and a following rotation by the angle $\gamma$ around the new $z$-axis $\left(z^{\prime}\right)$. See Fig. 3(a). Consequently, for three water molecules, the state space is 12-dimensional.

According to the distance-angle criterion, ${ }^{18,66}$ an $\mathrm{H}$-bond exists if the distance between two oxygen atoms $\mathrm{O}^{*}$ and $\mathrm{O}^{\mathrm{a}}$ is $R<0.35 \mathrm{~nm}$ and the angle between the $\overline{\mathrm{O}^{*} \mathrm{H}^{*}}$ and $\overline{\mathrm{O}^{*} \mathrm{O}^{\mathrm{a}}}$ vectors is $\angle\left(\overline{\mathrm{O}^{*} \mathrm{O}^{\mathrm{a}}}, \overline{\mathrm{O}^{*} \mathrm{H}^{*}}\right)<30^{\circ}$. There are four ways in which two water molecules can establish an H-bond; see Fig. 3(c) for our color coding which we use throughout this paper. In the green and cyan configuration, $\mathrm{O}^{\mathrm{a}}$ accepts an $\mathrm{H}$-bond from $\mathrm{O}^{*}$, whereas in the orange and red configuration, $\mathrm{O}^{\mathrm{a}}$ donates an $\mathrm{H}$-bond to $\mathrm{O}^{*}$ via $\mathrm{H}_{1}^{\mathrm{a}}$ and $\mathrm{H}_{2}^{\mathrm{a}}$, respectively.

\section{SUPPLEMENTARY MATERIAL}

See supplementary material for details on the Markov state model, the derivation of transition paths, the estimate of diffusivity profiles, and results for a restricted two-water Markov state model.

\section{ACKNOWLEDGMENTS}

This work was funded by the Deutsche Forschungsgemeinschaft (DFG) by Grant Nos. SFB 1114/C02 and SFB 1114/A04 and by the ERC consolidator Grant No. 772230. Discussions with Peter Hamm and Andrey Shalit are gratefully acknowledged.

${ }^{1}$ P. Ball, Chem. Rev. 108, 74 (2008).

${ }^{2}$ Y. M. Rhee, E. J. Sorin, G. Jayachandran, E. Lindahl, and V. S. Pande, Proc. Natl. Acad. Sci. U. S. A. 101, 6456 (2004).

${ }^{3}$ K. F. Rinne, J. C. F. Schulz, and R. R. Netz, J. Chem. Phys. 142, 215104 (2015).

${ }^{4}$ P. Setny, R. Baron, P. M. Kekenes-Huskey, J. A. McCammon, and J. Dzubiella, Proc. Natl. Acad. Sci. U. S. A. 110, 1197 (2013).

${ }^{5}$ A. Erbas, D. Horinek, and R. R. Netz, J. Am. Chem. Soc. 134, 623 (2012).

${ }^{6}$ R. Buchner and G. Hefter, Phys. Chem. Chem. Phys. 11, 8984 (2009).

${ }^{7}$ K. F. Rinne, S. Gekle, and R. R. Netz, J. Phys. Chem. A 118, 11667 (2014).

${ }^{8}$ D. Marx, M. E. Tuckerman, J. Hutter, and M. Parrinello, Nature 397, 601 (1999).

${ }^{9}$ D. Marx, ChemPhysChem 7, 1848 (2006).

${ }^{10}$ A. Hassanali, F. Giberti, J. Cuny, T. D. Kühne, and M. Parrinello, Proc. Natl. Acad. Sci. U. S. A. 110, 13723 (2013).

${ }^{11}$ X. Huang, C. J. Margulis, and B. J. Berne, Proc. Natl. Acad. Sci. U. S. A. 100, 11953 (2003).

${ }^{12}$ S. Sharma and P. G. Debenedetti, Proc. Natl. Acad. Sci. U. S. A. 109, 4365 (2012).

${ }^{13}$ A. Luzar, J. Chem. Phys. 113, 10663 (2000).

${ }^{14}$ D. Laage, G. Stirnemann, F. Sterpone, R. Rey, and J. T. Hynes, Annu. Rev. Phys. Chem. 62, 395 (2011).

${ }^{15}$ P. Debye, J. Soc. Chem. Ind. 48, 1036 (1929).

${ }^{16}$ F. S. Csajka and D. Chandler, J. Chem. Phys. 109, 1125 (1998).

${ }^{17}$ F. H. Stillinger, Science 209, 451 (1980).

${ }^{18}$ D. Laage, Science 311, 832 (2006).

${ }^{19}$ D. Laage and J. T. Hynes, J. Phys. Chem. B 112, 14230 (2008).
${ }^{20}$ D. Laage, J. Phys. Chem. B 113, 2684 (2009).

${ }^{21}$ K. Amann-Winkel, M.-C. Bellissent-Funel, L. E. Bove, T. Loerting, A. Nilsson, A. Paciaroni, D. Schlesinger, and L. Skinner, Chem. Rev. 116, 7570 (2016).

${ }^{22}$ H. J. Bakker and J. L. Skinner, Chem. Rev. 110, 1498 (2010).

${ }^{23}$ S. T. Roberts, K. Ramasesha, and A. Tokmakoff, Acc. Chem. Res. 42, 1239 (2009).

${ }^{24}$ J. D. Eaves, J. J. Loparo, C. J. Fecko, S. T. Roberts, A. Tokmakoff, and P. L. Geissler, Proc. Natl. Acad. Sci. U. S. A. 102, 13019 (2005).

${ }^{25}$ H. S. Chung, J. M. Louis, and W. A. Eaton, Proc. Natl. Acad. Sci. U. S. A. 106, 11837 (2009).

${ }^{26}$ S. Chaudhury and D. E. Makarov, J. Chem. Phys. 133, 034118 (2010).

${ }^{27}$ W. K. Kim and R. R. Netz, J. Chem. Phys. 143, 224108 (2015).

${ }^{28}$ C. J. Fecko, J. D. Eaves, J. J. Loparo, A. Tokmakoff, and P. L. Geissler, Science 301, 1698 (2003).

${ }^{29}$ C. J. Fecko, J. J. Loparo, S. T. Roberts, and A. Tokmakoff, J. Chem. Phys. 122, 054506 (2005).

${ }^{30}$ M. L. Cowan, B. D. Bruner, N. Huse, J. R. Dwyer, B. Chugh, E. T. J. Nibbering, T. Elsaesser, and R. J. D. Miller, Nature 434, 199 (2005).

${ }^{31}$ L. D. Marco, M. Thämer, M. Reppert, and A. Tokmakoff, J. Chem. Phys. 141, 034502 (2014).

${ }^{32}$ M. Heyden, J. Sun, S. Funkner, G. Mathias, H. Forbert, M. Havenith, and D. Marx, Proc. Natl. Acad. Sci. U. S. A. 107, 12068 (2010).

${ }^{33}$ J. Stenger, D. Madsen, P. Hamm, E. T. J. Nibbering, and T. Elsaesser, J. Phys. Chem. A 106, 2341 (2002).

${ }^{34}$ K. Liu, J. D. Cruzan, and R. J. Saykally, Science 271, 929 (1996).

${ }^{35}$ W. T. S. Cole and R. J. Saykally, J. Chem. Phys. 147, 064301 (2017)

${ }^{36}$ J. O. Richardson, C. Pérez, S. Lobsiger, A. A. Reid, B. Temelso, G. C. Shields, Z. Kisiel, D. J. Wales, B. H. Pate, and S. C. Althorpe, Science 351, 1310 (2016).

${ }^{37}$ Y. Mu, P. H. Nguyen, and G. Stock, Proteins: Struct., Funct., Bioinf. 58, 45 (2004).

${ }^{38}$ M. Ernst, F. Sittel, and G. Stock, J. Chem. Phys. 143, 244114 (2015).

${ }^{39}$ J.-H. Prinz, H. Wu, M. Sarich, B. Keller, M. Senne, M. Held, J. D. Chodera, C. Schütte, and F. Noé, J. Chem. Phys. 134, 174105 (2011).

${ }^{40}$ An Introduction to Markov State Models and Their Application to Long Timescale Molecular Simulation, Advances in Experimental Medicine and Biology, edited by G. R. Bowman, V. S. Pande, and F. Noé (Springer Heidelberg, 2014), Vol. 797.

${ }^{41}$ M. Sarich and C. Schütte, Metastability and Markov State Models in Molecular Dynamics, Courant Lecture Notes (American Mathematical Society, 2013).

${ }^{42}$ F. Noé, C. Schütte, E. Vanden-Eijnden, L. Reich, and T. R. Weikl, Proc. Natl. Acad. Sci. U. S. A. 106, 19011 (2009).

${ }^{43}$ V. A. Voelz, M. Jäger, L. Zhu, S. Yao, O. Bakajin, S. Weiss, L. J. Lapidus, and V. S. Pande, Biophys. J. 100, 515a (2011).

${ }^{44}$ S. Gu, D.-A. Silva, L. Meng, A. Yue, and X. Huang, PLoS Comput. Biol. 10, e1003767 (2014).

${ }^{45}$ F. Paul, C. Wehmeyer, E. T. Abualrous, H. Wu, M. D. Crabtree, J. Schöneberg, J. Clarke, C. Freund, T. R. Weikl, and F. Noé, Nat. Commun. 8, 1095 (2017).

${ }^{46}$ K. M. Thayer, B. Lakhani, and D. L. Beveridge, J. Phys. Chem. B 121, 5509 (2017).

${ }^{47}$ N. Plattner and F. Noé, Nat. Commun. 6, 7653 (2015).

${ }^{48}$ D. Shukla, Y. Meng, B. Roux, and V. S. Pande, Nat. Commun. 5, 3397 (2014).

${ }^{49}$ P. Hamm, J. Chem. Phys. 145, 134501 (2016).

${ }^{50}$ C. Gu, H.-W. Chang, L. Maibaum, V. S. Pande, G. E. Carlsson, and L. J. Guibas, BMC Bioinf. 14, S8 (2013).

${ }^{51}$ I. M. Svishev and P. G. Kusalik, Chem. Phys. Lett. 215, 596 (1993).

${ }^{52}$ Y. von Hansen, F. Sedlmeier, M. Hinczewski, and R. R. Netz, Phys. Rev. E 84, 051501 (2011).

${ }^{53}$ M. K. Scherer, B. Trendelkamp-Schroer, F. Paul, G. Perez-Hernandez, M. Hoffmann, N. Plattner, C. Wehmeyer, J.-H. Prinz, and F. Noé, J. Chem. Theory Comput. 11, 5525 (2015).

${ }^{54}$ A. Luzar and D. Chandler, Nature 379, 55 (1996).

${ }^{55}$ A. Luzar and D. Chandler, Phys. Rev. Lett. 76, 928 (1996).

${ }^{56}$ Y. L. A. Rezus and H. J. Bakker, J. Chem. Phys. 123, 114502 (2005).

${ }^{57}$ C. Zhang and G. Galli, J. Chem. Phys. 141, 084504 (2014).

${ }^{58}$ I. Popov, P. B. Ishai, A. Khamzin, and Y. Feldman, Phys. Chem. Chem. Phys. 18, 13941 (2016).

${ }^{59}$ D. C. Elton, Phys. Chem. Chem. Phys. 19, 18739 (2017).

${ }^{60}$ X.-Z. Li, B. Walker, and A. Michaelides, Proc. Natl. Acad. Sci. U. S. A. 108, 6369 (2011). 
${ }^{61}$ S. Habershon, T. E. Markland, and D. E. Manolopoulos, J. Chem. Phys. 131, 024501 (2009).

${ }^{62}$ V. S. Pande, K. Beauchamp, and G. R. Bowman, Methods 52, 99 (2010).

${ }^{63}$ H. J. C. Berendsen, J. R. Grigera, and T. P. Straatsma, J. Chem. Phys. 91, 6269 (1987)
${ }^{64}$ B. Hess, C. Kutzner, D. van der Spoel, and E. Lindahl, J. Chem. Theory Comput. 4, 435 (2008).

${ }^{65}$ H. J. C. Berendsen, J. P. M. Postma, W. F. van Gunsteren, A. DiNola, and J. R. Haak, J. Chem. Phys. 81, 3684 (1984).

${ }^{66}$ R. Kumar, J. R. Schmidt, and J. L. Skinner, J. Chem. Phys. 126, 204107 (2007). 